\title{
A new scenario applying traffic flow analogy to poleward expansion of auroras
}

\author{
Osuke Saka \\ Office Geophysik, Ogoori, Japan \\ Correspondence: Osuke Saka (saka.o@ nifty.com)
}

Received: 22 July 2018 - Discussion started: 28 August 2018

Revised: 24 February 2019 - Accepted: 21 May 2019 - Published: 5 June 2019

\begin{abstract}
Transient westward electric fields from the magnetosphere generate equatorward plasma drifts of the order of kilometers per second in the auroral ionosphere. This flow channel extends in north-south directions and is produced in the initial pulse of $\mathrm{Pi} 2$ pulsations associated with the field line dipolarization. Drifts in the ionosphere of the order of kilometers per second that accumulated plasmas at the lowlatitude end of the flow channel are of such large degree that possible vertical transport effects (including precipitation) along the field lines may be ignored. In this condition, we suggest that plasma compression in the ionosphere initiated the dynamic ionosphere. The dynamic ionosphere includes a nonlinear evolution of the compressed ionospheric plasmas, generation of field-aligned currents to satisfy the quasi-neutrality of the ionosphere, and parallel potentials associated with the excitation of an ion acoustic wave. We will study how the dynamic ionosphere created auroral expansion.
\end{abstract}

\section{Introduction}

"Auroras and solar corona observed at the solar eclipse are optical phenomena unique in space physics. With enough knowledge about the underlying physical processes, once auroras have been captured by a highly sensitive imager, they provide an unexpected wealth of information about plasma environment of the Earth" (Oguti, 2010). Plasma drifts in the ionosphere observed by the balloon-measured electric fields (Kelley et al., 1971), by the Ba releases (Haerendel, 1972), and by radar observations (Nielsen and Greenwald, 1978) that did not match the expanding trajectories of auroras were an example. They would have been observed in all-sky images as violent motion of auroras propagating poleward (Akasofu et al., 1966) or contact breakups initiated at the nearest approach to the hydrogen arc (Oguti, 1973). To account for the differences in propagation directions, it was suggested that the primary sources of auroral particles are in the magnetospheric plasmas, and they developed poleward in terms of propagation of rarefaction wave in the tail (Chao et al., 1977; Liu et al., 2012), tailward regression/braking of the fast earthward flows referred to as BBFs (Shiokawa et al., 1997; Haerendel, 2015), and onset instability of inner plasma sheet pressure (Nishimura et al., 2010). The above explanations were based on the observations that substorm expansion was initiated and amplified at the substorm onset by the BBFs arriving at the inner boundary of the plasma sheet from the tail (Kepko et al., 2004; Angelopoulos et al., 2008; Machida et al., 2009).

We show that the electric fields in the dipolarization front (DF) that are embedded in the leading edge of BBFs (Runov et al., 2011) triggered the ballooning instability of the stretched flux tubes in the inner magnetosphere. As a result, the stretched field lines returned to the dipole-like configurations by producing the convection surge. Westward electric fields associated with the convection surge were transmitted into the auroral ionosphere and yield the compressibility in the auroral ionosphere. The compressibility initiates the dynamic ionosphere and leads to an alternative scenario of the poleward expansion of auroras that we discuss in this paper. In this paper, field line reconfiguration and associated auroral breakups at dipolarization onset will be summarized in Sect. 2. In Sect. 3, we will show that the auroral ionosphere becomes compressive transiently during dipolarization. Section 4 will discuss generation of an ion acoustic wave for creating parallel potentials in the topside ionosphere. This 
contributes to the outflow. Poleward expansion of discrete auroras will be discussed in Sect. 5 in terms of a nonlinear evolution of the accumulated plasmas in the ionosphere. In the final section (Sect. 6), we summarize our results and apply the dynamic ionosphere to the nonconjugate auroras.

\section{Auroras and field line reconfiguration associated with geomagnetic $\mathrm{Pi} 2$ pulsation}

Poleward expansion of auroras arising out of the onset arc was observed in the initial pulse of Pi2 pulsations (Saka et al., 2012). Statistical study of field line inclinations at geosynchronous orbit for the intervals from $120 \mathrm{~min}$ prior to the Pi2 onset $(T-120)$ to $60 \mathrm{~min}$ after the onset $(T+60)$ is presented in Fig. 1 (reproduced from Saka et al., 2010). The inclination is measured positive northward from the $D-V$ plane of the $H V D$ coordinates. $H$ is positive northward parallel to dipole axis, $V$ is radial outward, and $D$ is dipole east. It shows that field line inclination at geosynchronous orbit (Goes5/6 at 285/252 ${ }^{\circ}$ in geographic coordinates) decreased continuously in the growth phase and attained minimum inclination angles, 33.6/49.4 ${ }^{\circ}, 2 \mathrm{~min}$ before the initial peak of Pi2 amplitudes. These inclination angles are smaller than $57.5 / 63.8^{\circ}$ estimated by the IGRF (International Geomagnetic Reference Field) model but rather fit the T89 model (Tsyganenko, 1989) for $\mathrm{Kp}=4\left(34.2 / 45.0^{\circ}\right)$. These field lines at the geosynchronous altitudes can be mapped to the auroral ionosphere at $63.4 / 62.7^{\circ} \mathrm{N}$ in geomagnetic coordinates by $\mathrm{T} 89$ for $\mathrm{Kp}=4$. Following the Pi2 onset, field line inclination turned to increase in a step-like manner at Goes5 while at Goes6, which is closer to the equatorial plane than Goes5, transient dipolarization pulses were observed. From these observations, we postulate that transient electric fields in DF triggered the ballooning instability of the stretched flux tubes at the arrival of BBFs. As a result, field lines turned back to dipole-like configurations by producing the convection surge. The convection surge may be observed by the geosynchronous satellites as the convection enhancement of the plasma sheet electrons due to local breakdown of the last open trajectories of plasma sheet electrons (Thomsen et al., 2002). The surge occurred in all-sky images coincident with the onset of bead-like rippling that leads to the breakups at the equatorward latitudes (Saka et al., 2014). In the subsequent $\mathrm{Pi} 2$ pulses, an auroral surge was observed in all-sky images between 66 and $74^{\circ} \mathrm{N}$ in geomagnetic latitudes, referred to as a poleward boundary aurora surge (PBAS) (Saka et al., 2012). They propagated eastward or westward at the poleward boundary of the auroral zone and were interpreted as an auroral manifestation of flow bifurcation of BBFs. In this onset scenario, the field line dipolarization finished in the initial pulse of the Pi2 pulsations and increased field line inclination in a step-like manner or generated dipolarization pulses. The convection surge occurred once in the initial pulse of BBFs (DF) but was not repeated in the following pulses in the BBF train. This correlation suggests that auroral breakup may not repeat in the Pi2 wave packet but occurs at its initial pulse.

\section{Horizontal plasma flows in the ionosphere}

We assume that westward electric fields associated with the convection surge were transmitted along the field lines to the auroral ionosphere by the guided poloidal mode (Radoski, 1967). The electric fields would be amplified during the projection into the ionosphere over $100 \mathrm{mV} \mathrm{m}^{-1}$ and created an equatorward flow through $\boldsymbol{E} \times \boldsymbol{B}$ drift of the order of kilometers per second in the auroral ionosphere. The flows would be confined in a flow channel expanding north-south in the midnight sector. The low-latitude end of the flow channel was at the latitudes of the onset arc. The high-latitude end may not expand beyond the poleward boundary of auroral zone. Longitudinal width of the flow channel may form a streamer (e.g., Nishimura et al., 2010) and develops after the breakups in about 1 to $2 \mathrm{~h}$ of local time $\left(\sim 1000 \mathrm{~km}\right.$ along $\left.65^{\circ} \mathrm{N}\right)$ corresponding to the horizontal-scale size of plasma flow vortices associated with Pi2 (Saka et al., 2014).

In the flow channel, drift across the magnetic fields for the $j$ th species $\left(\boldsymbol{U}_{j \perp}\right)$ can be written in the F region as (Kelley, 1989)

$\boldsymbol{U}_{j \perp}=\frac{1}{B}\left[\boldsymbol{E}-\frac{k_{\mathrm{B}} T_{j}}{q_{j}} \frac{\nabla n}{n}\right] \times \hat{\boldsymbol{B}}$.

Here, $\boldsymbol{E}$ denotes westward electric fields in the flow channel and $\hat{\boldsymbol{B}}$ denotes a unit vector of the magnetic fields $B$, downward in the auroral ionosphere. Symbols $k_{\mathrm{B}}, T_{j}, q_{j}$, and $n$ are the Boltzmann constant, temperature of the $j$ th species, charge of the $j$ th species, and density of electrons (ions), respectively. The electric field of the order of $100 \mathrm{mV} \mathrm{m}^{-1}$ exceeded the diffusion (second term) by 3 orders of magnitude in the low-temperature ionosphere. The $\boldsymbol{E} \times \boldsymbol{B}$ drift predominated in the $\mathrm{F}$ region and the diffusion term may be ignored. In the E region, drift trajectories may be written (Kelley, 1989) for electrons by

$\boldsymbol{U}_{\mathrm{e} \perp}=\frac{1}{B}[\boldsymbol{E} \times \hat{\boldsymbol{B}}]$

and for ions by

$\boldsymbol{U}_{\mathrm{i} \perp}=b_{\mathrm{i}}\left[\boldsymbol{E}+\kappa_{\mathrm{i}} \boldsymbol{E} \times \hat{\boldsymbol{B}}\right]$.

Here, $b_{\mathrm{i}}$ is the mobility of ions defined as $\Omega_{\mathrm{i}} /\left(B v_{\text {in }}\right)$, and $\kappa_{\mathrm{i}}$ is defined as $\Omega_{\mathrm{i}} / \nu_{\mathrm{in}}$. Symbols $\Omega_{\mathrm{i}}$ and $\nu_{\mathrm{in}}$ are ion gyrofrequency and ion-neutral collision frequency, respectively. $\hat{\boldsymbol{B}}$ denotes a unit vector of the magnetic fields $B$. To derive Eqs. (2) and (3), pressure gradient term (diffusion) was again ignored. In the $\mathrm{E}$ region $\left(\kappa_{\mathrm{i}}=0.1\right)$, plasma accumulation in equatorward latitudes by the imposed westward electric fields was produced by Eq. (2) for electrons and the second term in Eq. (3) for ions. Electrons smoothly moved equatorward while ions stopped in the original place because of low 

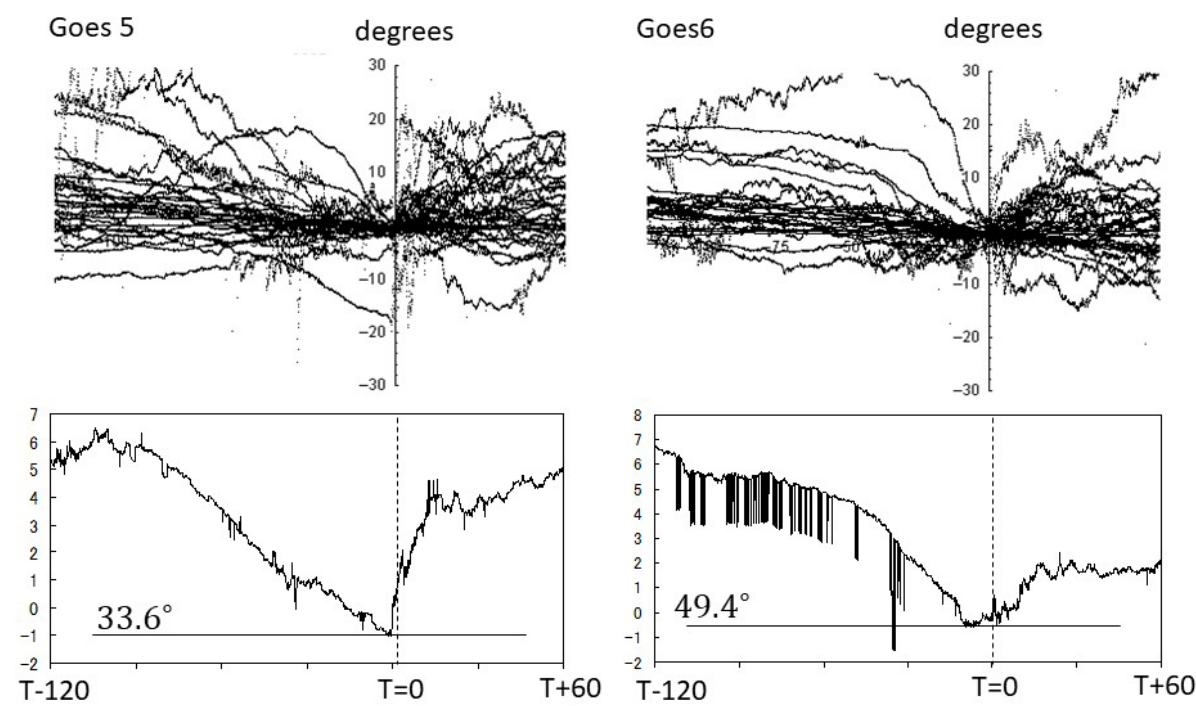

Figure 1. Inclination angles in degrees measured positive northward from the $V-D$ plane from 120 min prior to the Pi2 onset $(T-120)$ and to $60 \mathrm{~min}$ after the Pi2 onset $(T+60)$ reproduced from Saka et al. (2010). Magnetometer data of Goes 5/6 were represented in $H V D$ coordinates: $H$ is positive northward parallel to dipole axis, $V$ is radial outward, and $D$ is dipole east. Epoch superposition of 30 Pi2 events and mean angles calculated from them are plotted in top and in lower panels, respectively. Mean inclination angle at 2 min before the initial peak of Pi2 amplitudes $(T=0)$ was $33.6^{\circ}$ for G5 and $49.4^{\circ}$ for G6 in dipole coordinate. Dipolarization was step-like at Goes5, while at Goes 6 it was composed of dipolarization pulses. The average satellite latitudes estimated by $\mathrm{T} 89$ model were 10.3 and $7.9^{\circ} \mathrm{N}$ for Goes 5 and Goes6, respectively.

mobilities caused by high ion-neutral collisions. However, electron accumulation in lower latitudes increased southward electric fields and simultaneously ion drifts in the first term of Eq. (3) start. If the southward electric fields grew to exceed the westward electric fields by an order of magnitude, ion drifts in the first term of Eq. (3) and electron drifts in Eq. (2) balanced to satisfy the quasi-neutrality. This is equivalent to the generation of the Pedersen currents in the ionosphere. Thus, quasi-neutral electrostatic potential is generated in the $\mathrm{E}$ region, positive in poleward and negative in equatorward. The Pedersen currents would have closed to the field-aligned current (FAC), upward from the negative potential region and downward into the positive potential region to sustain the steady-state electrostatic potential in the ionosphere. Plasma drifts in the ionosphere, both in $\mathrm{E}$ and $\mathrm{F}$ regions, create a cavity in the high-latitude end of the flow channel and density pileup at the low-latitude end of the flow channel. We will focus on the density accumulation in the flow channel and discuss vertical transport of these accumulated materials. The development of the cavity in the flow channel may be the subject of another paper.

\section{Vertical plasma flows in the ionosphere}

A transient compression of the ionospheric plasmas at the low-latitude edge of the flow channel would excite the ion acoustic wave in the ionosphere traveling along the field lines in the upward and downward directions from the density peak of the $\mathrm{F}$ region. Figure 2 shows altitude distribution of the pre-onset density profile of electrons (black) and doubled density profile caused by the accumulation in red. The electron density profile in black was plotted using the sunspot maximum condition in the nightside given in Prince Jr. and Bostick Jr. (1964). The traveling ion acoustic waves, upward and downward, are denoted by vertical arrows. Ion acoustic wave propagating downward may be eventually absorbed in the neutrals, while the upward wave may propagate along the field lines further upward. We will focus only on the upwardtraveling ion acoustic wave. Electron motions produced the parallel electric fields in accordance with the Boltzmann relation (Chen, 1974),

$$
E_{/ /}=-\frac{k_{\mathrm{B}} T_{\mathrm{e}}}{q} \frac{\nabla_{/ / n_{\mathrm{e}}}}{n_{\mathrm{e}}} .
$$

Here, $k_{\mathrm{B}}$ is the Boltzmann constant, $q$ is electron charge, $T_{\mathrm{e}}$ is electron temperature, and $n_{\mathrm{e}}$ is electron density $\left(n_{\mathrm{e}}=n_{\mathrm{i}}\right)$. Equation (4) gives electric field strengths of the order of 0.4 and $2.0 \mu \mathrm{V} \mathrm{m}^{-1}$ for $T_{\mathrm{e}}=1000 \mathrm{~K}$ and $T_{\mathrm{e}}=5000 \mathrm{~K}$, respectively, when the $e$-folding distance of density dropout along the filed lines was $200 \mathrm{~km}$. For ions, steady-state motions exist in the ionosphere in the altitudes where ion-neutral collision frequencies exceed ion acoustic wave frequencies. In that case, parallel motions can be written as (Kelley, 1989)

$V_{\mathrm{i} / /}=b_{\mathrm{i}} E_{/ /}-D_{\mathrm{i}} \frac{\nabla_{/ / n}}{n}-\frac{g}{v_{\mathrm{in}}}$. 


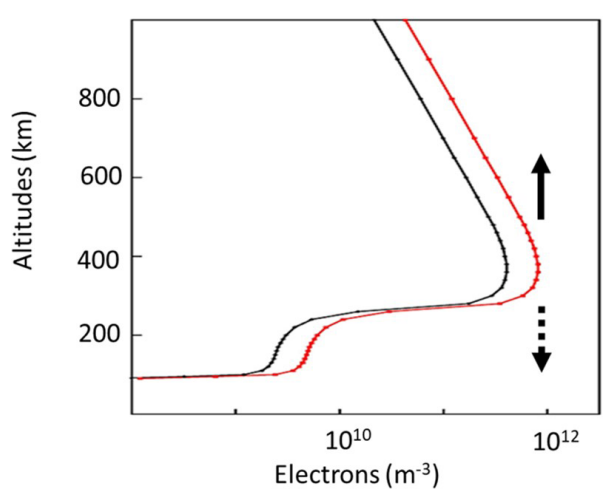

Figure 2. Vertical profiles from 90 to $1000 \mathrm{~km}$ in altitudes of electron number density in two conditions: pre-onset in black and after accumulation in red. The nighttime sunspot maximum condition given in Prince Jr. and Bostick Jr. (1964) was used to plot the preonset condition. Vertical arrows directing upward and downward denote traveling ion acoustic waves propagating along the field lines from the density peak of the $\mathrm{F}$ layer.

Here, $b_{\mathrm{i}}$ and $D_{\mathrm{i}}$ denote the mobility and diffusion coefficient of ions defined by $\frac{q_{\mathrm{i}}}{M_{\mathrm{i}} v_{\mathrm{in}}}$ and $\frac{k_{\mathrm{B}} T_{\mathrm{i}}}{M_{\mathrm{i}} \nu_{\mathrm{in}}}$, respectively. The symbols $M_{\mathrm{i}}, q_{\mathrm{i}}, v_{\mathrm{in}}$, and $g$ are ion mass, electric charge of ions, ionneutral collision frequency, and gravity, respectively. Ionneutral collision frequencies from 400 to $1000 \mathrm{~km}$ in altitudes were plotted in Fig. 3 using the nighttime sunspot maximum condition in Prince Jr. and Bostick Jr. (1964). Frequencies of ion acoustic wave were calculated by substituting the wavelength of ion acoustic wave into the dispersion relation. The wavelength was assumed to be identical to initial accumulation distance along the field lines. We chose two cases of 1000 and $4000 \mathrm{~km}$. Phase velocity of the ion acoustic wave of the order of $1600 \mathrm{~m} \mathrm{~s}^{-1}$ for the electron temperatures of $5000 \mathrm{~K}$ yields the wave frequencies of $1.6 \times 10^{-3} \mathrm{~s}^{-1}$ for the wavelength of $1000 \mathrm{~km}$ and $4.0 \times 10^{-4} \mathrm{~s}^{-1}$ for $4000 \mathrm{~km}$. These frequencies were overlaid in Fig. 3. Steady-state ion motions can be adopted up to $800 \mathrm{~km}$ for a wavelength over $1000 \mathrm{~km}$.

Altitude profiles of steady-state ion flows were evaluated substituting $1000 \mathrm{~K}$ for ion temperatures and the same $e$ folding distance in Eq. (4). The ions are oxygen and parallel electric fields are given by the Eq. (4). A snapshot of the velocity profile in altitudes from 400 to $800 \mathrm{~km}$ is shown in Fig. 4 for the two cases of electron temperatures: $5000 \mathrm{~K}$ for black dots and $1000 \mathrm{~K}$ for red dots. For the low-temperature case $(1000 \mathrm{~K})$, no ion upflow occurred because the parallel electric fields could not overcome gravity. We suggest that electron temperatures over $2700 \mathrm{~K}$ would be needed to excite ion upflow. When electron temperature was set to $5000 \mathrm{~K}$, the ion velocity of $15 \mathrm{~m} \mathrm{~s}^{-1}$ at $400 \mathrm{~km}$ in altitudes increased rapidly to $1369 \mathrm{~m} \mathrm{~s}^{-1}$ at $800 \mathrm{~km}$. The altitude profile of the flow velocity in Fig. 4 matched type-2 ion outflow observed by the EISCAT radar (Wahlund et al., 1992). We conclude that the ion upflow in topside ionosphere was caused pri-

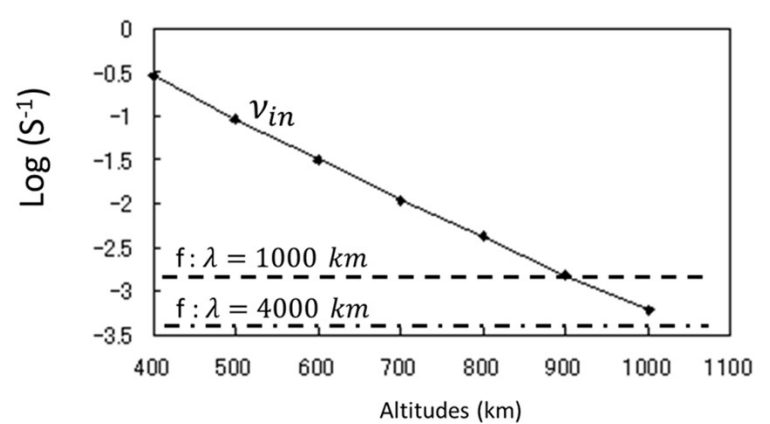

Figure 3. Ion-neutral collision frequency $\left(v_{\text {in }}\right)$ in altitudes from 400 to $1000 \mathrm{~km}$ calculated using the nighttime sunspot maximum condition in Prince Jr. and Bostick Jr. (1964). Wave frequencies of ion acoustic wave are overlaid for two wavelengths: 1000 and $4000 \mathrm{~km}$ along field lines (see text).

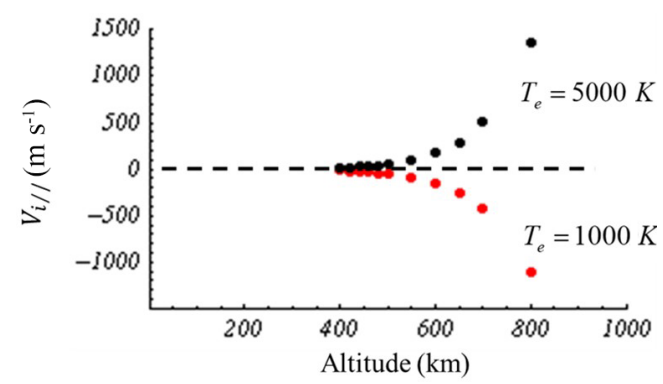

Figure 4. Steady-state parallel velocity in altitudes for ions (oxygen) produced by parallel electric fields: $0.4 \mu \mathrm{V} \mathrm{m}^{-1}\left(T_{\mathrm{e}}=1000 \mathrm{~K}\right)$ in red dots and $2.0 \mu \mathrm{V} \mathrm{m}^{-1}\left(T_{\mathrm{e}}=5000 \mathrm{~K}\right)$ in black dots. Vertical flows in altitudes from 400 to $800 \mathrm{~km}$ are shown. Flow velocity is positive upward and negative downward.

marily by the parallel electric fields excited by the upwardtraveling ion acoustic wave. Below $600 \mathrm{~km}$ in altitudes, upflow velocity was 1 to 2 orders of magnitude smaller than the equatorward drift in the flow channel. Upflow velocity became comparable to the horizontal drift over $800 \mathrm{~km}$ in altitudes and exceeded the phase velocity of ion acoustic wave. Ion outflow velocity exceeding the phase velocity of ion acoustic wave along the field lines may excite a shock at the topside ionosphere. A part of them developed to ion acoustic double layers (Sato and Okuda, 1980; Hasegawa and Sato, 1982; Hudson et al., 1983; Ergun et al., 2002) which were observed at the altitudes of $6000-8000 \mathrm{~km}$ (Mozer et al., 1977; Temerin et al., 1982). Those ion acoustic double layers would have produced parallel potential structures referred to as inverted- $V$-type electric fields.

\section{Nonlinear evolution of the horizontal flows}

Accumulation of electrons and ions occurred at the equatorward end of the flow channel. We can estimate a rate of accumulation by the following relation: 


$$
\frac{\Delta n}{\Delta t}=-n_{0} \frac{\Delta U}{\Delta x} .
$$

Here $n$ is plasma density, and $U$ denotes drift velocity in the flow channel in $x$. Substituting $\Delta U=10^{3} \mathrm{~m} \mathrm{~s}^{-1}$ and $\Delta x=10^{4} \mathrm{~m}$, we have $\frac{\Delta n}{\Delta t}=10^{10} \mathrm{~m}^{-3} \mathrm{~s}^{-1}$ for the background densityn $n_{0}=10^{11} \mathrm{~m}^{-3}$. This gives a density pileup of the order of $\frac{\Delta n}{n_{0}}=100 \%$ in $10 \mathrm{~s}$. If the equatorward drift in the flow channel is an order of $10^{3} \mathrm{~m} \mathrm{~s}^{-1}\left(E=100 \mathrm{mV} \mathrm{m}^{-1}\right.$ in the auroral ionosphere) and electron production by the precipitation does not exceed the accumulation rate, which was $100 \%$ of the background density in $10 \mathrm{~s}$, both outflows and precipitation may not bring significant changes to the flux carried by $E \times B$ drift in the flow channel. We then approximate one-dimensional (along the drift path in $x$ ) conservation equation in the flow channel.

$$
\frac{\partial n}{\partial t}+\frac{\partial}{\partial x}(n U)=0
$$

A question arises regarding maximum accumulation of plasmas at the equatorward end of the flow channel. One possible mechanism to suppress accumulation may be associated with the ionospheric screening that decreased the amplitudes of penetrated (total) westward electric fields by the increasing ionospheric conductivities. In a two-dimensional ionosphere with uniform height-integrated conductivity, total electric fields $E$ given by a sum of the incident $\left(E_{\mathrm{i}}\right)$ and reflected westward electric fields may be written as $E=\left(2 \Sigma_{\mathrm{A}}\left(\Sigma_{\mathrm{A}}+\Sigma_{\mathrm{P}}\right)\right) E_{\mathrm{i}}$, where $\Sigma_{\mathrm{A}}$ and $\Sigma_{\mathrm{P}}$ are the Alfven conductance defined by $1 / \mu_{0} V_{\mathrm{A}}$ and height-integrated Pedersen conductance in the ionosphere, respectively (Kan et al., 1982). Symbols $\mu_{0}$ and $V_{\mathrm{A}}$ denote magnetic permeability in vacuum and Alfven velocity, respectively. The amplitude ratio of total electric fields to incident electric fields is a function of the conductance ratio of Pedersen and Alfven; $E / E_{\mathrm{i}}=2$ for a low conductivity of the ionosphere satisfying $\Sigma_{\mathrm{P}} / \Sigma_{\mathrm{A}} \ll 1$, and $E / E_{\mathrm{i}}=0$ for a high conductivity of the ionosphere satisfying $\Sigma_{\mathrm{P}} / \Sigma_{\mathrm{A}} \gg 1$. Noting that $\Sigma_{\mathrm{P}}$ is proportional to the plasma density in the ionosphere, the total electric fields monotonically decreased with increasing plasma densities caused by accumulation itself and by the precipitations associated with the auroral activity. Another explanation may be suggested in the polarization electric fields (eastward) produced by the accumulation itself. These electric fields grew quickly with density accumulation and decreased the incident electric fields (westward) by the superposition. In addition to the above scenarios, we surmise that excess accumulation of the ionospheric plasmas may be suppressed through the term $(\boldsymbol{U} \cdot \nabla) \boldsymbol{U}$ in the equation of motion. From the ionospheric screening process discussed above, we tentatively assume that flow velocity $U$ is a function of the density $n$. Then the conservation Eq. (7) may be written as

$$
\frac{\partial n}{\partial t}+\frac{\partial}{\partial x} Q(n)=0
$$

Here, $Q(n)$ is a mass flux defined by $Q(n)=n U(n)$. This relation can be reduced to the nonlinear wave equation,

$$
\frac{\partial n}{\partial t}+c(n) \frac{\partial n}{\partial x}=0 .
$$

Here $c(n)$ is a wave propagation velocity defined by $c(n)=$ $U(n)+n U^{\prime}(n), U(n)$ is a drift velocity in the flow channel, and $U^{\prime}(n)$ denotes braking/acceleration of the drift velocity by increasing and decreasing density. The Eq. (9) is often referred to as propagation of kinematic waves to describe traffic flow (Lighthill and Whitham, 1955). In the following, we use dimensionless units normalized by $U_{m}$ and $n_{m}$. Here, $U_{m}$ and $n_{m}$ denote maximum drift velocity at $n=0$ and maximum density for complete stops of the drift, respectively. Assuming a constant braking in the flow channel, we define $U$ by a linear function of density $n$ as $U(n)=1-n$. Noting that $Q^{\prime}(n)=c(n)$, this relation is reduced to the equation $Q(n)=n(1-n)$, which is identical to the case for the traffic flow (Whitham, 1999). Both the $U$ and $Q$ are plotted in Fig. 5a as a function of $n$. A nonlinear evolution of the density waves is presented in Fig. $5 \mathrm{~b}$ by the characteristic curves. In the case of vehicles in traffic, the initial flows started from $n=0$ and stopped at $n=1.0$ by the tailback of cars. For the case of the ionosphere, the ionospheric density started from a finite density, $n=0.3$ in Fig. $5 \mathrm{~b}$, and increased to $n=1.0$ to terminate the flow by the full screening. The nonlinear evolution of the density profile in time is shown in Fig. $5 \mathrm{~b}$ in colors from black $\left(T=T_{1}\right)$, red $\left(T=T_{2}\right)$, green $\left(T=T_{3}\right)$, blue $\left(T=T_{4}\right)$, and to purple $\left(T=T_{5}\right)$. After $T=T_{5}$, the waves propagate upstream (poleward) as a shock. The shock velocity, $V$, is given as (Whitham, 1999)

$V=\frac{Q\left(n_{2}\right)-Q\left(n_{1}\right)}{n_{2}-n_{1}}$.

Here, subscript 1 is for the values ahead of shock and subscript 2 is for the values behind. Noting that $Q\left(n_{2}\right)=0$ and substituting $Q\left(n_{1}\right)=n_{1}\left(n_{2}-n_{1}\right)$, the Eq. (10) can be reduced to $V=-n_{1}$ in dimensionless unit. The propagation velocity of the shock is related to the densities ahead. For the case of $n=0.3$ in Fig. 5b, shock velocity can be estimated to be $-0.3 U_{m}$. Here, $U_{m}$ denotes maximum drift velocity in the ionosphere where ionospheric screening effects vanished by the condition $\Sigma_{\mathrm{P}} / \Sigma_{\mathrm{A}} \ll 1$. The shock velocity may be of the order of kilometers per second, which is comparable but opposite to the equatorward drift in the flow channel.

\section{Summary and discussion}

We proposed that the localized electric field drift introduced compressibility in the auroral ionosphere, which in turn generated field-aligned currents in the ionosphere for the quasineutrality, ion acoustic wave for parallel acceleration, and auroral expansions by nonlinear evolution of the ionospheric 
(a)

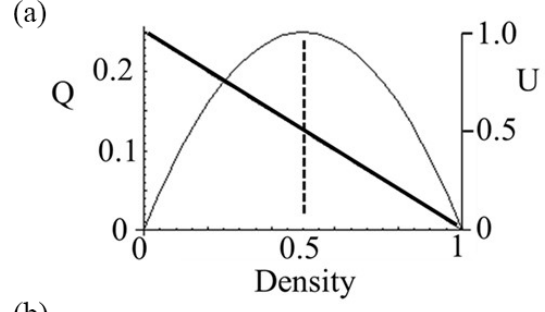

(b)

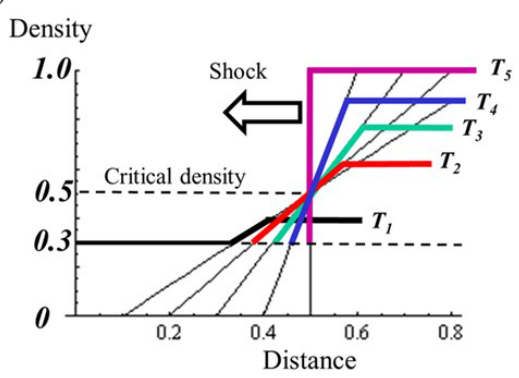

Figure 5. (a) Normalized flux ( $Q$ )-density ( $n$ ) curve (thin curve) and velocity $(U)$-density $(n)$ line (thick line) in flow channel. Vertical scale of the $U-n$ line is shown to the right; the scale of the $Q-n$ curve is to the left. Dotted line at $n=0.5$ indicates the critical density where $c(n)$ vanishes; waves are stationary relative to the ground. Waves propagate forward/backward at a density below/above the critical density. (b) Nonlinear evolution of the density accumulation. Density increased in a step-like manner from $T_{1}$ to $T_{5}$.

compression. We called the compressive ionosphere a dynamic ionosphere.

We apply this dynamic ionosphere to describe the asymmetry of discrete auroras in sunlit and dark hemispheres in the nightside sector (nonconjugate auroras). We suggest that the imbalance of the Pedersen conductance leads to the nonconjugate auroras: Pedersen conductance in the sunlit ionosphere is larger than that in the dark hemisphere. Larger Pedersen conductance or weaker electric fields in the sunlit ionosphere would have caused a weaker compressibility from which ion acoustic wave may not be excited or excited with only weak parallel potentials. This condition may reduce the occurrence probability of the discrete auroras and average energy of precipitating electrons in the sunlit hemisphere as exemplified in Newell et al. (1996) and Liou et al. (2001). Weaker electric fields in the sunlit ionosphere may also require a longer interval to accumulate enough plasmas to excite ion acoustic wave. Such an instance is described in Sato et al. (1998) where onset of auroral breakups in the sunlit ionosphere delayed that in the dark ionosphere.

Finally, we note that poleward expansion as described here is an auroral event occurring in the initial pulse of Pi2 pulsations. In the succeeding pulses in the Pi2 wave trains, auroras are composed of poleward surge propagating at the poleward boundary of auroral zone (PBAS) (Saka et al., 2012). We suppose that PBASs may be directly correlated to the re- connection processes inherent in the plasma sheet. This topic may be the subject of another paper.

Data availability. No data sets were used in this article.

Competing interests. The author declares that there is no conflict of interest.

Acknowledgements. The author would like to express his sincere thanks to all the members of the Global Aurora Dynamics Campaign (GADC) (Oguti et al., 1988). We also gratefully acknowledge the STEP Polar Network (http://center.stelab.nagoya-u.ac.jp/ cawses/datact/datact8.html, last access: May 2019). Geomagnetic coordinates and footprints of the satellites are available at the Data Center for Aurora in NIPR (http://polaris.nipr.ac.jp/ aurora, last access: November 2018).

Review statement. This paper was edited by Matina Gkioulidou and reviewed by two anonymous referees.

\section{References}

Akasofu, S.-I., Kimball, D. S., and Meng, C.-I.: Dynamics of the aurora-VII, Equatorward motions and the multiplicity of auroral arcs, J. Atmos. Terr. Phys., 28, 627-635, 1966.

Angelopoulos, V., McFadden, J. P., Larson, D., Carlson, C. W., Mend, S. B., Frey, H., Phan, T., Sibeck, D. G., Glassmeier, K.H., Auster, U., Donovan, E., Mann, I. R., Rae, I. J., Russell, C. T., Runov, A., Zhou, X.-Z., and Kepko, L.: Tail reconnection triggering substorm onset, Science, 321, 931-935, 2008.

Chao, J. K., Kan, J. R., Lui, A. T. Y., and Akasofu, S.-I.: A model for thinning of the plasma sheet, Planet. Space Sci., 25, 703-710, 1977.

Chen, F. F.: Introduction to plasma physics, Plenum Press, New York, 1974.

Ergun, R. E., Andersson, L., Main, D., Su, Y.-J., Newman, D. L., Goldman, M. V., Carlson, C. W., McPhadden, J. P., and Mozer, F. S.: Parallel electric fields in the upward current region of the aurora: Numerical solutions, Phys. Plasmas, 9, 3695-3704, 2002.

Haerendel, G.: Plasma drifts in the auroral ionosphere derived from Barium release, in: Earth magnetospheric processes, edited by: McComac, B. M., D.Reidel Publishing Company, DordrechtHolland, 246-257, 1972.

Haerendel, G.: Substorm onset: Current sheet avalanche and stop layer, J. Geophys. Res., 120, 1697-1714, https://doi.org/10.1002/2014JA020571, 2015.

Hasegawa, A. and Sato, T.: Existence of a negative potential solitary-wave structure and formation of a double layer, Phys. Fluids, 25, 632-635, 1982.

Hudson, M. K., Lotko, W., Roth, I., and Witt, E.: Solitary waves and double layers on auroral field lines, J. Geophys. Res., 88, 916-926, 1983. 
Kan, J. R., Longenecker, D. U., and Olson, J. V.: A transient response model of Pi2 pulsations, J. Geophys. Res., 87, 74837488, 1982.

Kelley, M. C.: The earth's ionosphere: plasma physics and electrodynamics, Academic Press, Inc., San Diego, California, 1989.

Kelley, M. C., Starr, J. A., and Mozer, F. S.: Relationship between magnetospheric electric fields and the motion of auroral forms, J. Geophys. Res., 76, 5256-5277, 1971.

Kepko, L., Kivelson, M. G., McPherron, R. L., and Spence, H. E.: Relative timing of substorm onset phenomena, J. Geophys. Res., 109, A04203, https://doi.org/10.1029/2003JA010285, 2004.

Lighthill, M. J. and Whitham, G. B.: On kinematic waves. II. A theory of traffic flow on long crowded roads, P. Roy. Soc. Lond. A Mat., 229, 317-345, 1955.

Liou, K., Newell, P. T., and Meng, C.-I.: Seasonal effects on auroral particle acceleration and precipitation, J. Geophys. Res., 106, 5531-5542, 2001.

Liu, W. W., Liang, J., Donovan, E. F., and Spanswick, E.: If substorm onset triggers tail reconnection, what triggers substorm onset, J. Geophys. Res., 117, A11220, https://doi.org/10.1029/2012JA018161, 2012.

Machida, S., Miyashita, Y., Ieda, A., Nosé, M., Nagata, D., Liou, K., Obara, T., Nishida, A., Saito, Y., and Mukai, T.: Statistical visualization of the Earth's magnetotail based on Geotail data and the implied substorm model, Ann. Geophys., 27, 1035-1046, https://doi.org/10.5194/angeo-27-1035-2009, 2009.

Mozer, F. S., Carlson, C. W., Hudson, M. K., Torbert, R. B., Parady, B., Yatteau, J., and Kelley, M. C.: Observations of paired electrostatic shocks in the polar magnetosphere, Phys. Rev. Lett., 38, 292-295, 1977.

Newell, P. T., Meng, C. I., and Lyons, K. M.: Suppression of discrete aurorae by sunlight, Nature, 381, 766-767, 1996.

Nielsen, E. and Greenwald, R. A.: Variations in ionospheric currents and electric fields in association with absorption spikes during substorm expansion phase, J. Geophys. Res., 83, 5645-5654, 1978.

Nishimura, Y., Lyons, L. R., Zou, S., Xing, X., Angelopoulos, V., Mende, S. B., Bonnell, J. W., Larson, D., Auster, U., Hori, T., Nishitani, N., Hosokawa, K., Sofko, G., Nicolls, M., and Heinselman, C.: Preonset time sequence of auroral substorms: Coordinated observations by all-sky imagers, satellites, and radars, J. Geophys. Res., 115, A00108, https://doi.org/10.1029/2010JA015832, 2010.

Oguti, T.: Hydrogen emission and electron aurora at the onset of the auroral breakup, J. Geophys. Res., 78, 7543-7547, 1973.

Oguti, T.: Introduction to auroral physics, Laboratory for SolarTerrestrial Environment, Nagoya University, 2010 (in Japanese).

Oguti, T., Kitamura, T., and Watanabe, T.: Global aurora dynamics campaign, 1985-1986, J. Geomagn. Geoelectr., 40, 485-504, 1988.
Prince, Jr., C. E. and Bostick Jr., F. X.: Ionospheric transmission of transversely propagated plane waves at micropulsation frequencies and theoretical power spectrums, J. Geophys. Res., 69, 3213-3234, 1964.

Radoski, H. R.: Highly asymmetric MHD resonances: The guided poloidal mode, J. Geophys. Res., 72, 4026-4027, 1967.

Runov, A., Angelopoulos, V., Zhou, X.-Z., Zhang, X.-J., Li, S., Plaschke, F., and Bonnell, J.: A THEMIS multicase study of dipolarization fronts in the magnetotail plasma sheet, J. Geophys. Res., 116, A05216, https://doi.org/10.1029/2010JA016316, 2011.

Saka, O., Hayashi, K., and Thomsen, M.: First $10 \mathrm{~min}$ intervals of $\mathrm{Pi} 2$ onset at geosynchronous altitudes during the expansion of energetic ion regions in the nighttime sector, J. Atmos. Sol.-Terr. Phy., 72, 1100-1109, 2010.

Saka, O., Hayashi, K., and Koga, D.: Periodic aurora surge propagating eastward/westward at poleward boundary of aurora zone during the first $10 \mathrm{~min}$ intervals of $\mathrm{Pi} 2$ onset, J. Atmos. Sol.-Terr. Phy., 80, 285-295, https://doi.org/10.1016/j.jastp.2012.02.010, 2012.

Saka, O., Hayashi, K., and Thomsen, M.: Pre-onset auroral signatures and subsequent development of substorm auroras: a development of ionospheric loop currents at the onset latitudes, Ann. Geophys., 32, 1011-1023, https://doi.org/10.5194/angeo32-1011-2014, 2014.

Sato, N., Nagaoka, T., Hashimoto, K., and Saemundsson, T.: Conjugacy of isolated auroral arcs and nonconjugate auroral breakups, J. Geophys. Res., 103, 11641-11652, 1998.

Sato, T. and Okuda, H.: Ion-acoustic double layers, Phys. Rev. Lett., 44, 740-743, 1980.

Shiokawa, K., Baumjohann, W., and Haerendel, G.: Braking of high-speed flows in the near-Earth tail, Geophys. Res. Lett., 24, 1179-1182, 1997.

Temerin, M., Cerny, K., Lotko, W., and Mozer, F. S.: Observations of double layers and solitary waves in the auroral plasma, Phys. Rev. Lett., 48, 1176-1179, 1982.

Thomsen, M. F., Korth, H., and Elphic, R. C.: Upper cutoff energy of the electron plasma sheet as a measure of magnetospheric convection strength, J. Geophys. Res., 107, SMP 25-1-SMP 25-18, https://doi.org/10.1029/2001JA000148, 2002.

Tsyganenko, N. A.: A magnetospheric magnetic field model with a warped tail current sheet, Planet. Space Sci., 37, 5-20, 1989.

Wahlund, J.-E., Opgenoorth, H. J., Haggstrom, I., Winser, K. J., and Jones, G. O.: EISCAT observations of topside ionospheric outflows during auroral activity: revisited, J. Geophys. Res., 97, 3019-3017, 1992.

Whitham, G. B.: Linear and nonlinear waves, A Wiley-Interscience Publication, John Wiley \& Sons, Inc., New York, 1999. 